\title{
Comparison of Various Outdoor Propagation Models with Empirical Data Collection using TEMS 10.0.5 for DIT University, Dehradun
}

\author{
Govind Sati \\ DIT University \\ Dehradun, Uttarakhand \\ India
}

\author{
Sonika Singh, Ph.D \\ DIT University \\ Dehradun, Uttarakhand \\ India
}

\begin{abstract}
An exact radio propagation model is important for coping up with new challenges in the field of communication like: appropriate design, deployment and service management strategies for any wireless network. Analysis of outdoor propagation models is important for the purpose of signal coverage prediction, data reception analysis of performance parameters like Rx Lev, Rx Qual, FER, BER, reception schemes, and analysis of signal attenuation under different environments and in the determination of the optimum location for the purpose of installation of base stations. The aim of this work is to carry out a comparative study of different outdoor propagation models with respect to empirical data collected from DIT University (formally Dehradun Institute of Technology), Dehradun by drive testing using TEMS Investigation tools at $1.8 \mathrm{GHz}$. This study involves obtaining the mean received power distribution at specified receiver distance from the respective GSM base station. The measurement process was based on signal strength measurement and the study revealed that out of all the outdoor propagation models. Only COST Hata model, COST Hata W-B model and Ericsson model approximated the measurement characteristics and hence they can be deployed for network planning in this region. Measurement process of signal strength and data collection methodology also discuss in this paper.
\end{abstract}

\section{Keywords}

Path loss, MapInfo, TEMS, Drive testing, Outdoor propagation models

\section{INTRODUCTION}

Radio system planning can be seen as s process that defines the stages required to provide a desired radio network plan for a given geographical area. To achieve a desired radio network, it is important to capture critical factors that influence the choice of base transceiver station locations. It is important to note that the parameters that affect radio system planning also define the radio propagation environment that specifies the characteristics of the radio propagation which furthermore has a significant effect on the coverage and capacity of radio network. [2]

\section{TEST SITE AND SCENARIO SELECTION}

In this paper the test site selected in DIT University located at Makkawala Greens, Po Bhagwantpur, Mussoorie Diversion Road, Dehradun, which is categorized under rural environment. Propagation characteristics of models depend on morphology and environment (like urban suburban and rural).
Region of the DIT University started form $500 \mathrm{~m}$ of the BTS station. Figure.1 showed the satellite view of DIT University.

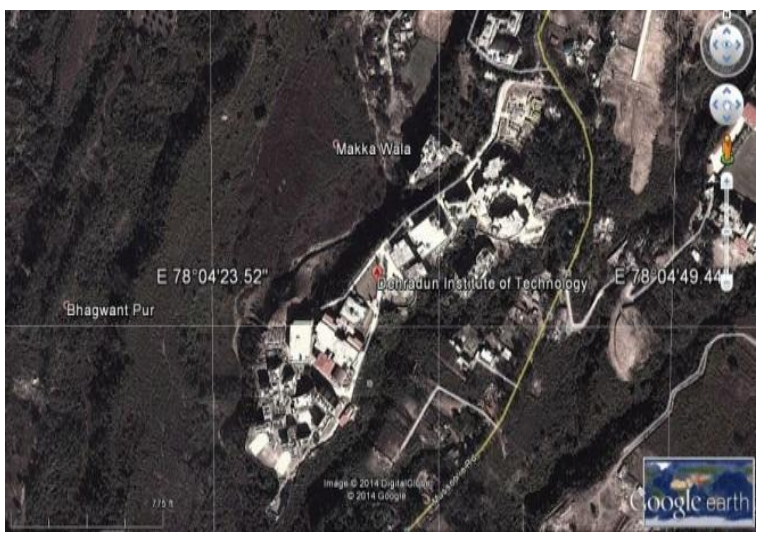

Fig.1 Satellite view of DIT-University.

Measurement of signal strength is based on the effect of density of vegetation and height of buildings in the test site. The height of the base station antenna, distance between transmitter and receiver represent the characteristics of test site. Base station antenna installed on the top of building or top of the mountain. Usually omnidirectional antennas are used for predesign drive test and the measurements are made all around the site. Figure.2. Shown the base state along with DIT University, Dehradun.

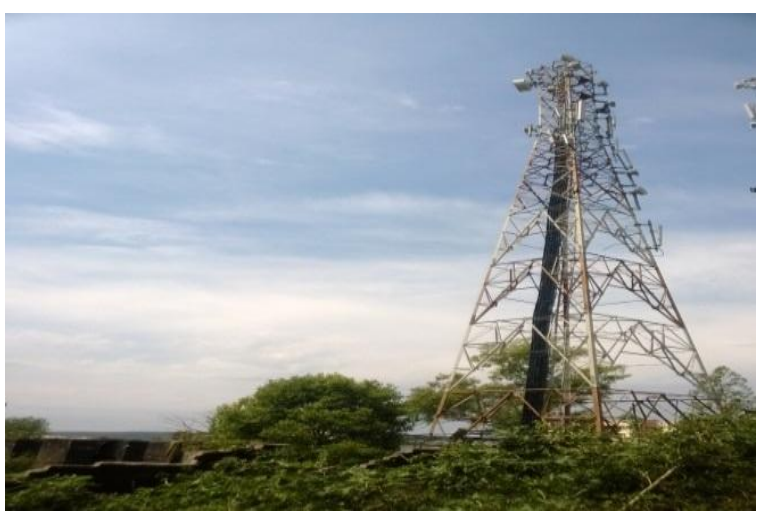

Fig.2 Radio signal base station along DIT-University, Dehradun.

When propagation model used in one site (e.g. suburban) is optimized based on the drive test on this site measurement, whereas the same model used for different site (e.g. rural) is adjusted based drive test for that sites. 


\section{DRIVE TEST MEASUREMENT AND DATA COLLECTION METHODOLOGY FOR DIT UNIVERSITY}

Measurement process started with a Hata's propagation models by default. These models are parametric model with some coefficients. Accuracy of predication (for a given environment) based on the correct choice of these coefficients. Main objective of drive testing is to provide real data based on test site of coefficient, which minimizes the prediction error. PlaNet and Cell CAD propagation tools provide these coefficients.

In field measurement we analyzed that signal variations is due to propagation and slow fading. The received signals are sampled over a spatial window, these window are called bins. Sampling rate, averaging window and number of bins to be measured are considered in sampling process.

The enough number of independent samples are to measure for each window. Maximum sampling rate $R s$ or minimum sampling interval in time is dependent on speed of vehicle during the drive testing. Whenever the vehicle is not moving the measurement process has to be stopped. We estimate the best set of correction factor to minimize the prediction errors by comparing the predicted and the measured signal strength on the drive testing routes.

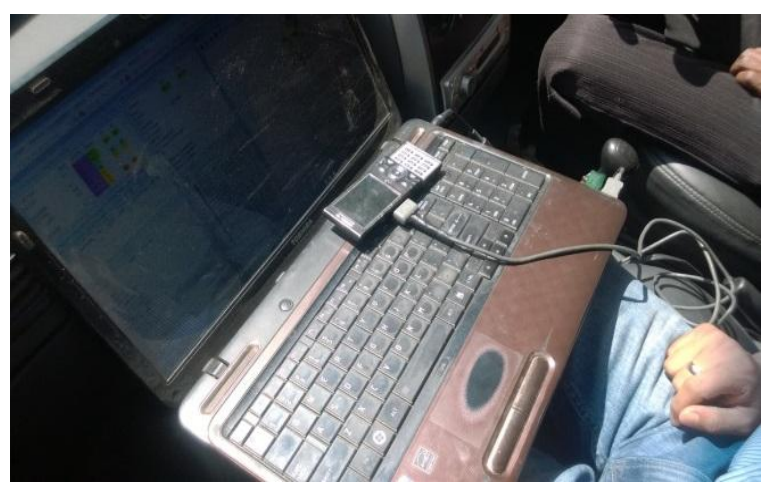

Fig.3 Process of data collection depicted using real-time drive test with TEMS Investigation.

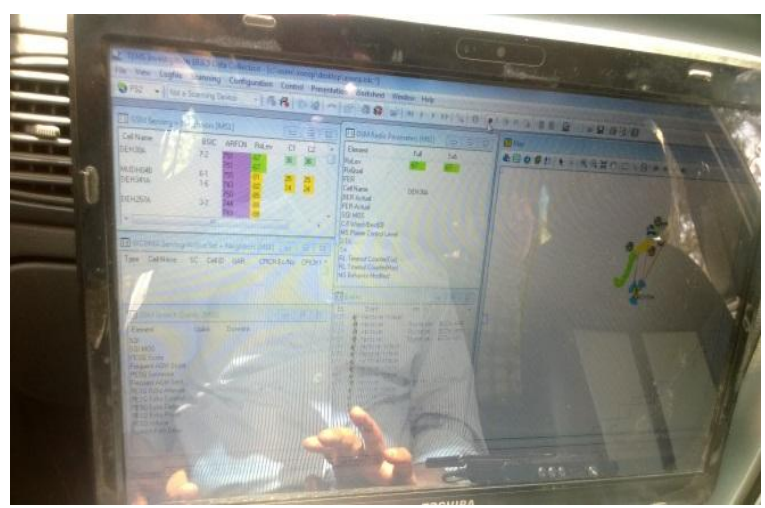

Fig.4 Process of data collection depicted using real-time drive test.

The methodology for data fetch deals with the drive test tools which contains both software and hardware devices. For drive test measurement for the region of DIT University TEMS
10.0.5 has been used. Drive test process contains TEMS Investigation, GPS, TEMS dongle, test mobile, power supply and vehicle. TEMS Investigation software is very important for data collection process. GPS systems, Dongle, test mobile also very important for drive testing these devices are connected to laptop via USB 2.0. USB provide interface between these devices with software. The GPS system connected with laptop via USB 2.0. GPS system determines the radial distance of mobile vehicle from the base station. TEMS dongle and test mobile are also connected to the laptop.

We can access TEMS Investigation software using HASP SRM security key. HASP SRM is offered in two versions, one using hardware-based and one using software-based protection keys. They are called "HASP SRM HL" (figure3.3) and "HASP SRM SL" respectively. Now the data collection process is started with mobile vehicle. In data collection process it is important for the vehicle to move continuously at the speed of 30 to $40 \mathrm{Kmh}$.

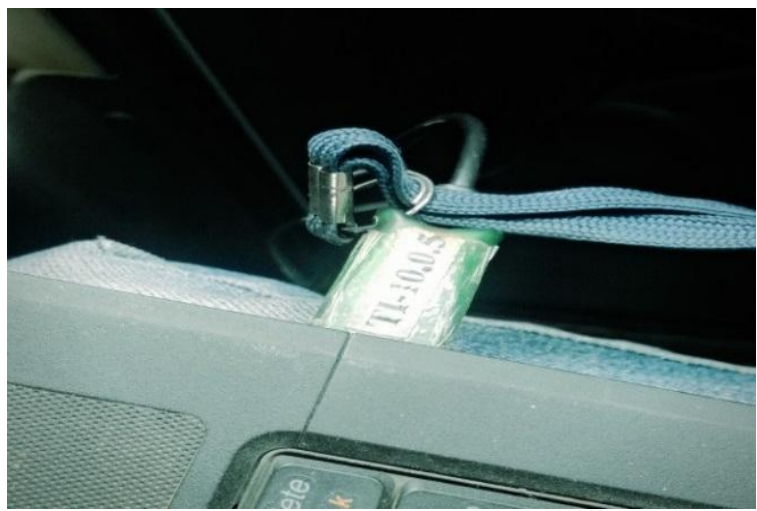

Fig.5 HASP SRM HL key (USB)

Mobile van drive around all the sectors of base station for the complete coverage of area and record local receive power.

\section{COMPARATIVE ANALYSIS AND METHODOLOGY OF OUTDOOR PROPAGATION MODELS FOR DIT UNIVERSITY, DEHRADUN REGION}

\subsection{Free Space Path Loss Models}

Path loss in free space can be defined as the ratio of the transmitted power to receiver power in free air. It is expressed in decibels. Free space path loss is diverse on frequency and distance. Path loss is expressed as [4]

$P L=10 n \log _{10}(d)+10 n \log _{10}(f)+32.44$

Where $\mathrm{n}$ is the path loss exponent. The value of path loss exponent for free space model is always 2.[3]

$P L=20 \log _{10}(d)+20 \log _{10}(f)+32.44$

$\mathrm{F}[\mathrm{MHz}]$ Frequency of operation.

$\mathrm{D}[\mathrm{m}]$ Distance between transmitter \& receiver. 


\subsection{STANFORD UNIVERSITY INTERIM MODEL (SUI)}

Working group of IEEE 108.16 proposed this standard for the frequency range below $11 \mathrm{GHz}$. The proposed standards for frequency range below $11 \mathrm{GHz}$ contain the channel models developed by Stanford University, namely the SUI models. This model has been derived as an extension to Hata model with $1900 \mathrm{MHz}$ frequency band and above.

The fundamental path loss expression for the SUI model along with correction factors is as [4]

$P L=A+10 \gamma \log _{10}\left(\frac{d}{d_{0}}\right)+X_{f}+X_{h}+e ;$ for $d>d_{0} \quad \ldots$ (3)

Where the parameters are

D $[\mathrm{m}]$ Separation of transmitter and receiver

$d_{0}[\mathrm{~m}] 100$

$\lambda \quad[\mathrm{m}]$ Wavelength

$X_{f}[\mathrm{MHz}]$ Correction factor for frequency $>2 \mathrm{GHz}$

$X_{h}[\mathrm{~m}]$ Correction factor for receiving antenna height

$\mathrm{S}$ [dB] Correction factor for shadowing effect (value between $8.2 \mathrm{~dB}$ to $10.6 \mathrm{~dB}$ )

$\gamma$ Path loss exponent

\subsection{COST 231 HATA MODEL}

Cost 231 Hata model is widely used for predicting path loss in mobile wireless system. Cost 231 Hata model is initiated as an extension of Hata model. It is used for predicted the path loss in different environments like urban, sub urban and rural. It is designed for $500 \mathrm{MHz}$ to $2000 \mathrm{MHz}$ frequency range. The basic path loss equation in $\mathrm{dB}$ is[4]

$P L=46.3+33.9 \log _{10}(f)-13.82 \log _{10}\left(h_{b}\right)-a h_{m}+$ $n\left(44.9-6.55 \log _{10}\left(h_{b}\right)\right) \log _{10} d+c_{m}$.(4)

Where,

f: [MHz] Frequency

$\mathrm{d}:[\mathrm{Km}]$ Distance between transmitter and receiver antenna

$h_{b}[\mathrm{~m}]$ Height of transmitter antenna

The parameter $a h_{m}$ is defined for urban environments as

$a h_{m}=3.20\left(\log _{10}\left(11.75 h_{r}\right)\right)^{2}-4.97$, for $f>400 M H z$

And for suburban or rural environments

$a h_{m}=\left(1.1 \log _{10} f-0.7\right) h_{r}-\left(1.56 \log _{10} f-0.8\right)$

Where

$h_{r}$ Antenna height in meters.

\subsection{METHODOLOGY}

Continuous wave (CW) signals are transmitted be base station antenna. Omnidirectional antenna is used in the base station. This omnidirectional antenna has azimuth pattern and gain of $8.14 \mathrm{dBi}$ [1]. Omnidirectional antenna mounted on the top of base station. This is to ensure that the BTS station sites used are performing optimally and meets up with all parameters. [5]

BTS power: $45 \mathrm{dBm}$

MS Antenna height: 2.5 to $4 \mathrm{~m}$

Connector loss: $3 \mathrm{~dB}$

Feeder loss: $2.58 \mathrm{~dB}$

Duplexer loss: $4.5 \mathrm{~dB}$

MS Antenna gain: 14dBi

BTS Antenna gain: $18.3 \mathrm{dBi}$

EIRP $P_{t}: 67.22 \mathrm{dBm}$

Power conversion from Watt to $\mathrm{dBm}$ in done using the following expression:

$P_{d B m}=10 \log _{10} P_{m W} \ldots(5)$

Formula for effective power radiated from the BTS antenna $P_{t \text { in } d B m}$ is given as:

$P_{t}=P_{B T S}-P_{c o n}-P_{D}-P_{t}+\left(A_{m s}+A_{B T S}\right) \ldots$

Where $P_{B T S}=$ Base station power, $P_{D}=$ Duplexer loss, $P_{t}=$ Feeder loss, $A_{m s}=$ Mobile station (receiver) antenna gain, $A_{B T S}=$ Base antenna gain.

Power at the receiver distances from the base station is expressed as:

$P_{L}=P_{t}-P_{r}$ in $\mathrm{dBm}$

$P_{L}(d B)=10 \log _{10}\left(P_{t} / P_{r}\right)$

Using these equations we measured path loss values. The obtained values from field measurements are then compared with those calculated using the existing

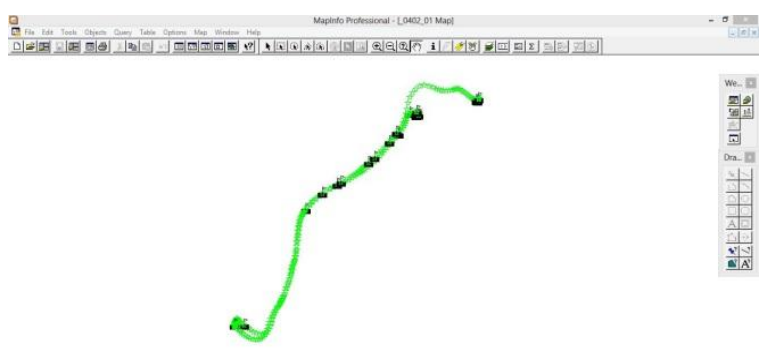

Fig.6 MapInfo plot of test site. 


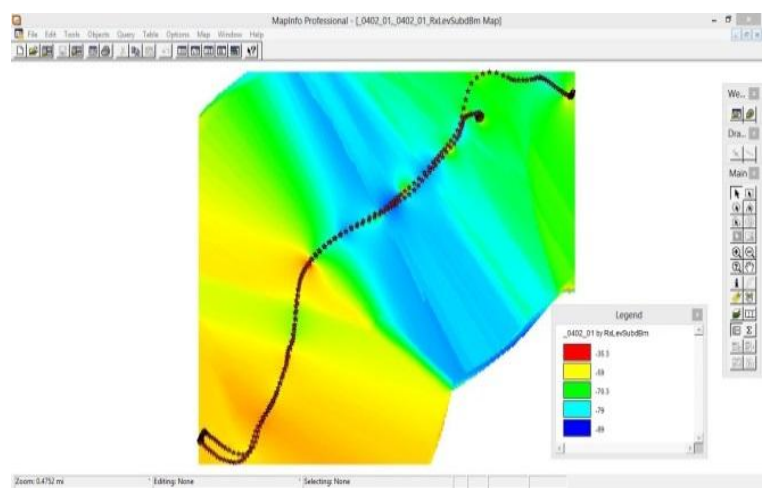

Fig.7 MapInfo plot of signal power transmission of the base station

\section{RESULTS}

DIT University selected for this experiment. The table below shows the received level power and path loss results obtained during the test drive.

\begin{tabular}{|c|c|c|}
\hline $\begin{array}{c}\text { Tx/Rx } \\
\text { Distance } \\
(\text { Km })\end{array}$ & Rx-level(dBm) & $\begin{array}{c}\text { Path-loss } \\
(\mathbf{d B m})\end{array}$ \\
\hline 0.5 & -70 & 137.22 \\
\hline 0.6 & -83 & 154.22 \\
\hline 0.7 & -83 & 154.22 \\
\hline 0.8 & -65 & 132.22 \\
\hline 0.9 & -47 & 113.22 \\
\hline 1.0 & -55 & 122.22 \\
\hline 1.1 & -57 & 124.22 \\
\hline 1.2 & -55 & 122.22 \\
\hline 1.3 & -84 & 155.22 \\
\hline 1.4 & -79 & 148.22 \\
\hline 1.5 & -74 & 144.22 \\
\hline
\end{tabular}

Table.1 Received level power and path loss.

This context under covers a comparative outlook on different propagation models for path los measurement [7]. It also compares pre-existing theoretical profiles with empirical measurement [6] (depicted measured values vs. distance) with different theoretical models singularly and in a cumulative fashion too. MATLAB R2010a used for simulation. Simulated result shown in following figure.

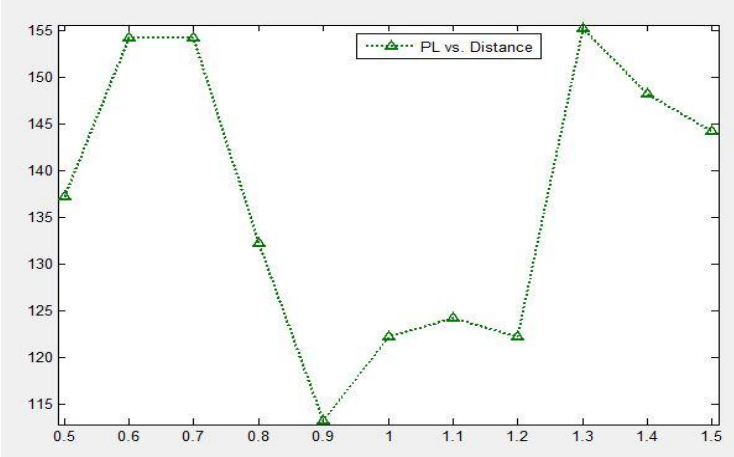

Fig.8 Distance vs. Measured path loss

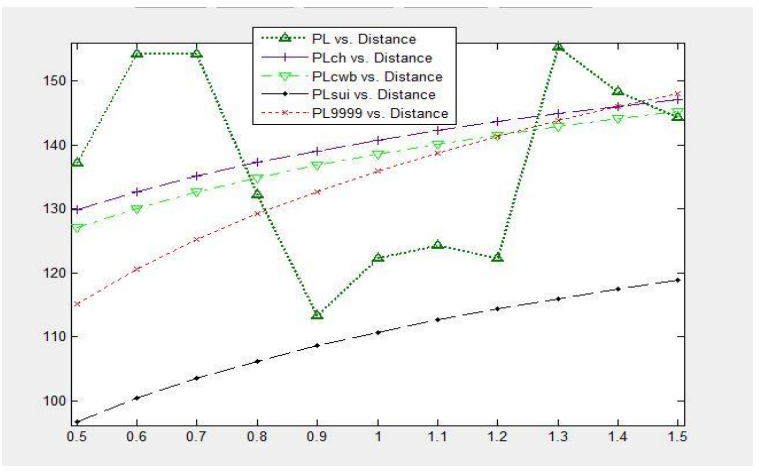

Fig.9 Comparison of different outdoor propagation models with measured path loss for DIT University.

Figure.9 showed the comparison of path loss of different outdoor propagation models with measured path loss. We considered path loss of Cost 231 Hata model, Cost 231 WB model, SUI model and Ericsson model 9999 for comparison. $\mathrm{X}$ axis shown the distance and $\mathrm{Y}$ axis show cross pounding path loss in above figure. Region of the DIT University started form $500 \mathrm{~m}$ of the BTS station.

\section{CONCLUSION}

Our comparison of different outdoor propagation models on basis of theoretical path loss with field measured data for DIT University, Dehradun. It was revealed during the comparative study of different models along with interpolated measured field data values that out of different models studied only three specific models closely approximate the actual measurement characteristics namely: COST Hata model, Ericsson model and COST W-B model. Apart from these three models all other models showed huge deviation from the measured field data value, which clearly indicates inefficiency of all other models to be deployed for network planning in this region (i.e. DIT University, Dehradun) at $1.8 \mathrm{GHZ}$ of operational frequency band.

\section{REFERENCES}

[1]. Vinko Erceg, Larry J. Greenstein, Sony Y. Tjandra, Seth R. Parkoff, Ajay Gupta, Boris Kulic, Arthur A. Julius and Rence Bianchi. IEEE Journal on selected areas in communication. Vol. 17. No. 7 July 1999. "An empirical based path loss models for wireless channels in suburban environments"

[2]. Ed. Upper Saddle River, NJ 07458 Prentice Hall PTR. 2002. Rapport T.S Wireless Communication Principles and Practice $2^{\text {nd }}$. 
[3]. Magdy F. Iskander, Zhengquing Yun, ' Propagation predication models for wireless communication systems', IEEE Transactions on microwave theory \& techniques, March 2012

[4]. Govind Sati, Sonika Singh. IJCES ISSN: 2231-6590, March 2014. A review on outdoor propagation models in radio communication.

[5]. Shoewu, O and F. O. Edeko, AJSIR ISSN 2153-649X, doi:10.5251/ajsir. 2011.2.3.438.455. Analysis of radio wave propagation in Lagos environs.

[6]. Technickivjesnik Josip Milanovic, Snjezana Rime Drlje and Lvo Majerski,17, UDC/UDK621.391.8:004.733 (497.13 Osijek) , ISSN: 1330-3651, 2010, Radio wave propagation mechanisms and empirical models for fixed wireless access systems,
[7]. Vishal Gupta, Sandip Vijay and S. C. Sharma, ISN2008, Feb. 22-24, 2008, Pg-271. Efficient Path Loss Prediction in Mobile Wireless Communication Network,

[8]. Hemant kumar sharama, Santosh Sahu, Sanjeev Sharma. IJCA (0975-8887). Vol. 19-No.6 April 2011., Enhanced Cost231 W.I. propagation model in wireless network.

[9]. Naren Mohan, Praven Chandrasekaran and Hossam Hmimy, 0-78903-9152-7105. 2005 IEEE. Drive test based EDGE radio network performance evolution.

[10]. Timo Nihtila. 978-1-4673-990-5112. 2012 IEEE, QoS verification for minimization of drive tests in LTE networks.Fedor Chernogorov. 